\title{
From Anemia to Erythropoietin Resistance in Head and Neck Squamous Cell Carcinoma Treatment: A Carousel Driven by Hypoxia
}

This article was published in the following Dove Press journal: OncoTargets and Therapy

\section{Grazia Lazzari (D) \\ Giovanni Silvano}

Radiation Oncology Unit, Saint Giuseppe Moscati Hospital, Taranto 74100, Italy
Correspondence: Grazia Lazzari; Giovanni Silvano

Radiation Oncology Unit, Saint Giuseppe Moscati Hospital, Road to Martina Franca, Taranto 74100, Italy

Tel +39-099-458572I; +39-099-458582 I

Fax +39-349 53008I 0; +39-3356547748

Email lazzarigrazia@gmail.com;

gisilva@tin.it

\begin{abstract}
Anemia has been identified as a significant negative prognosticator in head and neck squamous cell carcinoma (HNSCC) concurrent chemoradiotherapy (CCRT). Irrespective of the causes, anemia in HNSCC is believed to contribute to intratumoral hypoxia, which reduces the effectiveness of radiotherapy and oxygen-dependent chemotherapy. Correction of anemia with recombinant human erythropoietin (rHu-EPO) has been performed as a surrogate for hypoxia compensation to improve tumor control and survival outcomes. However, the results of the most important EPO clinical trials have been disappointing. Following the recent finding that EPO and its receptor (EPOR) are both expressed in HNSCC specimens, a new hypothesis has been advanced. This postulates that hypoxic signaling might activate EPOR through the hypoxia-inducible factor (HIF) signaling pathway and its downstream effectors, including carbonic anhydrase 9 (CA-9), glucose transporter 1 (GLUT-1), and vascular endothelial growth factor (VEGF), leading to the failure of rHu-EPO treatment, as assessed from the results of the best-known EPO trials. This review addresses the relationship among anemia, hypoxia, and tumoral EPO/EPOR expression in HNSCC treatment in an attempt to elucidate the main mechanisms involved in the resistance to $\mathrm{rHu}-\mathrm{EPO}$ therapy, as in a carousel.
\end{abstract}

Keywords: HNSCC, CCRT, recombinant human erythropoietin, EPO trials, hypoxiainducible factor

\section{Introduction}

Anemia is defined as a condition resulting from a reduced concentration of red blood cells or low hemoglobin $(\mathrm{Hb})$ levels in peripheral blood, which decreases the oxygen-carrying capacity of the blood and leads to tissue hypoxia. ${ }^{1}$ Although having a low hemoglobin level has been shown to be detrimental, what represents a low level is still undefined, with reported values ranging from $9-14.5 \mathrm{~g} / \mathrm{dL}$. Irrespective of the $\mathrm{Hb}$ value and causes, anemia in HNSCC patients is known to impair treatment effectiveness and negatively affect disease prognosis. ${ }^{3}$ For decades, anemia has been considered as a surrogate marker of tumor hypoxia. In turn, hypoxia is a recognized feature of radioresistance because patients with low $\mathrm{Hb}$ levels exhibit reduced locoregional tumor control and a low survival probability. ${ }^{4}$ Despite their heterogeneity, HNSCCs are considered an ideal model for assessing the relationship between anemia and tumor hypoxia in radiation therapy. ${ }^{5}$ To justify anemia correction with recombinant human erythropoietin ( $\mathrm{rHu}-\mathrm{EPO})$, it is reasonable to expect that treatment outcome would be better for well-oxygenated tumors 
than for poorly oxygenated ones. ${ }^{6,7}$ Indeed, the results of studies using animal models have suggested that rHu-EPO therapy may enhance tumor radiosensitivity. ${ }^{89}$ However, the most important EPO clinical trials assessing the role of rHu-EPO in concurrent chemoradiotherapy (CCRT) for HNSCC have failed in terms of local control and overall survival (OS). ${ }^{10-13}$ The finding that the erythropoietin receptor (EPOR) is expressed in several nonhematopoietic tissues and different cancer types, including breast, lung, prostate, and skin cancers, ${ }^{14}$ and is overexpressed in tongue and oral squamous cancer specimens, could explain the resistance to rHu-EPO therapy. ${ }^{15,16}$ These results have led to the hypothesis that rHu-EPO might act on the EPO receptor expressed in hypoxic tumors and enhance their malignant properties. This hypothesis postulates that hypoxic signaling might activate EPOR expression, as found in HNSCC specimens, through several signaling pathways, including the hypoxia-inducible factor (HIF) pathway and its downstream target proteins like carbonic anhydrase 9 (CA-9), glucose transporter 1 (GLUT-1), and vascular endothelial growth factor (VEGF) that are known to regulate tumor invasiveness in hypoxic and acidic environments. ${ }^{17}$ Indeed, elevated HIF and CA-9 expression have been associated with decreased survival in HNSCC patients treated with CCRT. ${ }^{18}$ Consequently, the negative results provided by the EPO trials should be considered the tip of an iceberg comprising the hypoxiarelated HIF signaling pathway in tumors. Here, we review the relationship among anemia, hypoxia, and tumoral EPOR expression in the resistance to $\mathrm{rHu}-\mathrm{EPO}$ therapy based on the most important published data and new advances in research on hypoxia-targeting agents and hypoxia new imaging assessment.

\section{Anemia in HNSCC and CCRT}

In HNSCC, the overall anemia-related mortality risk has been estimated at $75 \% .{ }^{19}$ It has been known for decades that anemia has a detrimental effect on tumor prognosis and treatment outcome, inducing hypoxia and thereby reducing the effectiveness of chemotherapy and radiotherapy. ${ }^{20}$ The pathophysiology of anemia in cancer is multifactorial, and includes cancer-related factors as well as treatment-related side effects.

\section{Anemia in HNSCC}

In HNSCC patients, anemia may be a direct consequence of malnutrition resulting from mechanical impairment of the ability to eat or swallow, as occurs in oro- hypopharyngeal cancers. Metabolic alterations affecting appetite and mucositis in the irradiated area may further enhance the swallowing impairments, thereby exacerbating the malnutrition, weight loss, and cancer cachexia. ${ }^{21}$ In turn, cancer cachexia is associated with complex metabolic, molecular, and cellular alterations related to both inflammatory and anticancer immune responses. ${ }^{22}$ The interaction between tumor cell populations and the immune system leads to the release of cytokines, especially interferon gamma, interleukin 1, and tumor necrosis factor, which disrupts endogenous EPO synthesis in the kidney and suppresses the differentiation of erythroid precursor cells in the bone marrow. Moreover, macrophage activation leads to a shorter erythrocyte half-life in the spleen and liver, as well as a decrease in iron utilization, resulting in anemia with high levels of EPO and/or syderopenic anemia with high levels of ferritin. ${ }^{23}$

\section{Anemia and Cisplatin Nephrotoxicity}

Anemia is the most common hematological side effect in cancer patients receiving chemotherapy, presenting with a mild or moderate severity in about $60 \%$ of cancer patients after platinum-based schedules. ${ }^{24,25}$ Cisplatin and other platinum-based compounds are alchilant cytotoxic drugs that can kill cancer cells by damaging DNA, inhibiting DNA synthesis and mitosis, and inducing apoptotic cell death. ${ }^{26}$ These effects are amplified with the addition of ionizing radiation, explaining why cisplatin is considered the most important radiosensitizing drug supplied in the CCRT protocols for HNSCCs in light of a toxicity profile that does not overlap with that of radiotherapy. Thus, cisplatin-CCRT has been validated as the standard care in locally advanced HNSCC based on the results of meta-analyses and randomized trials that have used different treatment schedules. ${ }^{27-30}$ The effective cumulative dose of cisplatin in CCRT protocols for HNSCC is significantly related to survival $(p=0.027)$, suggesting that the recommended cumulative dose of cisplatin to be administered during radiotherapy should be at least $200 \mathrm{mg} / \mathrm{m}^{2}{ }^{31}$ The kidney is the major route of cisplatin excretion, and accumulates cisplatin to a greater extent than other organs, with a concentration in proximal tubular epithelial cells approximately fivefold that its concentration in serum. This indicates that the disproportionate accumulation of cisplatin in kidney tissue contributes to cisplatin-induced nephrotoxicity by damaging renal proximal tubular cells through a decrease in endogenous EPO production. ${ }^{32}$ The kidney is the major source of EPO synthesis in the adult, 
and there is some evidence that EPO production occurs in peritubular interstitial cells, although some studies have suggested a renal tubular cell origin under hypoxic conditions. ${ }^{33}$ Several factors have been identified as underlying cisplatin-induced renal nephrotoxicity, such as the paradoxical role of gamma-glutamyl transpeptidases, the unexpected ability of proximal tubular cells to metabolize cisplatin to a nephrotoxin, and the roles of apoptotic pathways and reactive oxygen metabolites. ${ }^{32}$ Consequently, cisplatin-associated anemia has been defined as "an EPO deficiency syndrome" that rapidly responds to EPO administration, which, in most patients, corrects the anemia associated with renal failure. ${ }^{33}$

\section{The Hemoglobin Threshold for Anemia}

There is evidence that a strong correlation may exist between anemia and local control in HNSCC concurrent chemoradiotherapy. Indeed, a dose-effect relationship has been established for local control $(p=0.04)$ and survival $(p=0.04)$ when grouping by $\mathrm{Hb}$ concentration $(<120$, $120-140$, and $>140 \mathrm{~g} / \mathrm{L}$ ), demonstrating that anemia is strongly associated with these parameters. ${ }^{34}$ However, the precise $\mathrm{Hb}$ threshold value remains unresolved, with reported values ranging from $9-14.5 \mathrm{~g} / \mathrm{dL}^{2}$

\section{Prognostic Value of $\mathrm{Hb}$}

Several studies have investigated this question. In the DAHANCA II study, a strong correlation between pretreatment $\mathrm{Hb}$ levels and local control was noted in male patients with oro-pharyngeal tumors treated with misonidazole and exhibiting pretreatment $\mathrm{Hb}$ levels $\geq 14.5 \mathrm{~g} / \mathrm{dL}$. The five-year local tumor control rate was $61 \%$ as compared with only $14 \%$ in the placebo-treated patients who presented pretreatment $\mathrm{Hb}$ values $<14.5 \mathrm{~g} / \mathrm{dL}{ }^{35} \mathrm{~A}$ different study showed that patients who presented $\mathrm{Hb}$ values $>13.0 \mathrm{~g} / \mathrm{dL}$ had a significantly higher two-year locoregional tumor control and survival rates than those with $\mathrm{Hb}$ values $<13.0 \mathrm{~g} / \mathrm{dL}$ (95\% and $88 \%$ vs $66 \%$ and $46 \%$, respectively; $p=0.0018) .{ }^{36}$ These results were subsequently confirmed by Prosnitz et al, who obtained a significantly higher five-year, failure-free survival for patients with $\mathrm{Hb}$ levels $\geq 13 \mathrm{~g} / \mathrm{dL}$ compared with those with Hb values $<13 \mathrm{~g} / \mathrm{dL}(75 \%$ vs $50 \% ; p<0.01) .{ }^{37}$ Rades et al used the multivariate analysis to evaluate the outcome in stage IV HNSCC treated with CCRT, and found an improved OS associated with a $\mathrm{Hb}$ value $>12 \mathrm{~g} / \mathrm{dL}(p=0.048)$, and this value also seemed to be related to improved local control and metastasis- free survival $\left(p<0.001\right.$ and $p=0.012$, respectively). ${ }^{38}$ In agreement with these early studies, a more recent reassessment through a noninterventional, single-blinded, randomized study on advanced HNSCC confirmed that a preradiotherapy $\mathrm{Hb}$ level $>10.7 \mathrm{~g} / \mathrm{dL}$ was a prognosticator in locally advanced HNSCC patients treated with chemoradiation $(p<0.001)$. Hemoglobin levels $>10.7 \mathrm{~g} / \mathrm{dL}$ were associated not only with improved treatment outcome, but also with higher performance status, fewer treatment interruptions, and lesser degree of mucositis. ${ }^{39}$ Additionally, this question was reassessed in a postoperative CCRT study, which defined a low nadir $\mathrm{Hb} 11$ $\mathrm{g} / \mathrm{dL}$ with radiation treatment time $>7$ weeks as poor prognosticators related to a significantly higher risk of death (hazard ratio $=4.0 ; 95 \% \mathrm{CI}=1.6-10.2 ; p=0.004) .{ }^{40}$

\section{The Hb Threshold for Treatment with Erythropoiesis-Stimulating Agents}

The available guidelines regulating the administration of erythropoiesis-stimulating agents (ESAs) propose considerably different $\mathrm{Hb}$ threshold values for the use of these agents. According to the updated guidelines of the European Organization for Research and Treatment of Cancer (EORTC), a sustained hemoglobin level of approximately $12 \mathrm{~g} / \mathrm{dL}$ is the recommended target for treatment with ESAs to ameliorate symptoms, avoid transfusion therapy, and significantly improve the quality of life, mainly in patients with chemotherapy-induced anemia. ${ }^{41}$ However, the ASCO-ASH guidelines recommend ESA treatment for patients with chemotherapy-associated anemia whose cancer treatment is not curative in intent and whose $\mathrm{Hb}$ level has declined to $\leq 10 \mathrm{~g} / \mathrm{dL}^{42}$

\section{Anemia Correction with rHu-EPO}

Anemia correction through exogenous administration of $\mathrm{rHu}-\mathrm{EPO}$ has been suggested to improve treatment outcomes in HNSCC patients in terms of tumor control and quality of life. ${ }^{43}$ EPO, the main erythropoiesis-regulating hormone, is primarily synthesized in the kidney and liver under hypoxic conditions. ${ }^{44}$ The actions of EPO in the bone marrow are mediated by EPOR, the EPO-specific receptor located on red blood cell precursors. EPO/EPOR signaling exerts two major functions, i.e., it stimulates the proliferation of erythroid progenitor cells and maintains their viability. ${ }^{45}$ Interestingly, EPOR is also expressed in several nonhemopoietic tissues, as well as in different cancer types. $^{14-16}$ Although preclinical studies and clinical trials have explored the potential impact of $\mathrm{rHu}-\mathrm{EPO}$ treatment 
on tumor control and survival outcomes in CCRT-treated HNSCC, the results have been disappointing.

\section{Preclinical Studies}

Preclinical data have suggested that rHu-EPO may enhance tumor radiosensitivity and improve the clinical efficacy of radiation therapy and chemotherapy. ${ }^{7}$ Stüben et al demonstrated that $\mathrm{rHu}-\mathrm{EPO}$ restored radiosensitivity in experimental human tumors in anemic mice. ${ }^{8,9}$ In this model, the development of anemia in mice was inhibited by administration of $\mathrm{rHu}$-EPO beginning two weeks before anemia induction by total body irradiation (TBI) $(2 \times 4$ Gy) performed prior to tumor transplantation into the subcutis of the hind leg. Approximately two weeks after TBI (tumor volume of approximately $40 \mathrm{~mm}^{3}$ ), single-dose irradiation (12 Gy) of the tumor was performed, resulting in tumor growth delay. Radiation-induced anemia corresponding to a $\mathrm{Hb}$ concentration of $9.9 \mathrm{~g} / \mathrm{dL}$ led to reduced radiosensitivity when compared with controls. The radiation dose to control $50 \%$ of the tumors (TCD50) with rHu-EPO treatment for anemia prevention $(\mathrm{Hb}$ concentration $=13.3 \mathrm{~g} / \mathrm{dL})$ was 39.8 Gy (TCD50 = 49.4 Gy, vs 40.1 Gy for controls), indicating that radiosensitivity was restored when compared with anemic mice. Moreover, the prevention of anemia through $\mathrm{rHu}-\mathrm{EPO}$ treatment resulted in a significantly increased growth delay $(61 \pm 5$ days $)$ when compared with untreated anemic animals. ${ }^{46}$

\section{EPO Trials}

However, several EPO trials have yielded controversial results, with all having provided a gain in terms of the hemoglobin level, but not in terms of survival or tumor control. In a double-blind, randomized, placebo-controlled trial, Henke et al observed that patients given epoetin beta achieved $\mathrm{Hb}$ concentrations higher than $140 \mathrm{~g} / \mathrm{L}$ (women) or $150 \mathrm{~g} / \mathrm{L}$ (men) compared with patients given placebo. However, locoregional progression-free survival (LRPFS) was poorer with epoetin beta than with placebo (adjusted relative risk $1.62[95 \% \mathrm{CI}=1.22-2.14] ; p=0.0008)$. The relative risk was $1.69(1.16-2.47, p=0.007)$ for locoregional progression, and $1.39(1.05-1.84, p=0.02)$ for survival. ${ }^{10} \mathrm{In}$ the RTOG 99-03 trial, although $\mathrm{Hb}$ levels at 4 weeks rose by an average of $1.66 \mathrm{~g} / \mathrm{dL}$ in the EPO arm, compared with an average decrease of $0.24 \mathrm{~g} / \mathrm{dL}$ in the control arm $(p=0.0001)$, no significant difference was observed between the treatment arms in the locoregional failure (LRF) primary endpoint. The three-year LRF rate was $36 \%$ for the control and $44 \%$ for the EPO arm $(p=0.56)$. Additionally, no significant differences were recorded in LRPFS, patterns of failure, OS, or toxicity. The three-year LRPFS rate was $52 \%$ for the control and $47 \%$ for the EPO group, and the OS rate was $57 \%$ and $56 \%$, respectively. ${ }^{11}$ The epoetin alpha trial by Hoskins et al also provided disappointing results. Although $\mathrm{Hb}$ levels increased from baseline with epoetin alpha treatment, the median duration of local disease-free survival (DFS) was not significantly different between groups (observation, 35.42 months; epoetin alpha, 31.47 months). Moreover, there was no significant difference in DFS, OS, tumor outcome, or cancer treatmentrelated anemia or fatigue between groups. ${ }^{12}$ Additionally, the trial with darbepoetin also failed to show significant positive results in tumor control and survival. In the ENHANCE (Evaluation of NeoRecormon ${ }^{\circledR}$ (epoetin beta) on outcome in Head and Neck Cancer) trial, treatment with darbepoetin alpha increased the $\mathrm{Hb}$ level to the planned value in $81 \%$ of the patients. However, the results showed a poorer outcome, with a five-year cumulative LRF rate of $47 \%$ and $34 \%$ for the darbepoetin alpha and control arms, respectively. This was also seen for the endpoints of event-free survival, diseasespecific death, and OS. ${ }^{13}$ All these findings raise the question: if anemia influences hypoxia, does hypoxia play a role?

\section{Hypoxia and Tumors}

Although HNSCCs are heterogenous, they share several features that make them an ideal model from a radiobiological perspective to assess the effect of radiation therapy on anemia and tumor hypoxia. Tumor reoxygenation is one of the $5 \mathrm{Rs}$ of radiobiology, and anemia is considered to be a radioresistance factor as it contributes to tumor hypoxia. ${ }^{47}$ Two subtypes of hypoxia have been described, namely, acute and chronic. ${ }^{48}$ Acute hypoxia is a transient phenomenon that has been defined as either a local disturbance in perfusion due mainly to the loss of microvascular blood flow, or a strong variation in red blood cell flux that leads to a decreased microvascular oxygen supply to the tumor area due to an inadequate and aberrant neovasculature. When tumor growth is greater than the accompanying neovascularization, cancer cells furthest from the vessels that are at the limit of oxygen diffusion show hypoxic tension and tumor necrosis. This phenomenon is called chronic or diffusion-limited hypoxia and can activate stress response genes to compensate for hypoxemia. Hypoxic regions are defined as areas with oxygen tension (pO2) values $\leq 10 \mathrm{mmHg}$, and have been identified in several solid tumors, although mainly in HNSCCs. ${ }^{49}$ It is well known that hypoxic cells are markedly less sensitive to radiation than welloxygenated cells, owing to several mechanisms that directly and indirectly induce proteomic and genomic changes that 
polarize the tumor microenvironment. ${ }^{50}$ In turn, these effects contribute to increased tumor invasiveness and metastatic potential, loss of apoptosis, and chaotic angiogenesis, which further increase treatment resistance. Further tumor microenviroment is not static but a highly dynamic structure consisting of subpopulations of cancer cells that have been differentially exposed to hypoxia for different times (minutes or hours) and then reoxygenated again leading to a "cycling hypoxia" due to variations in red blood cells (RBCs) flux through an abnormal tumour vascularisation. ${ }^{51}$ Cycling hypoxia is a key determinant of radioresistance, impaired drug delivery and increased metastatic potential in HNSCC but also an important feature in the hypoxia imaging assessment.

\section{HPV-Associated HNSCC and Hypoxia}

HPV-associated HNSCC is a distinct entity due to Human papilloma virus (HPV) infection which has been detected especially in oropharyngeal tumors. ${ }^{52}$ It is characterized by a different molecular biology, epidemiology, radiosensivity and prognosis. ${ }^{53}$ Several clinical studies have explored the possible association between the favorable radiosensivity of HPV positive tumors and their hypoxic status with gene expression profiles assessment, $\mathrm{pO} 2$ measurements or hypoxia positron emission tomography (PET) scanning, ${ }^{54,55}$ but no positive correlation between HPV status and tumor hypoxia have been recorded, yielding disappointing results concerning the link of radiosensivity and hypoxia. In fact randomized trials investigating the effect of hypoxic modification as nimorazole and tirapazamine in HNSCC have shown no evaluable effects in HPV positive tumors. ${ }^{56,57}$ In vitro studies addressing this correlation in HPV-positive cell lines, have provided the evidence that HPV-positive cell lines are, overall, more radiosensitive than HPV-negative cell lines. ${ }^{58}$ However the effect of hypoxia and nimorazole during irradiation has been demonstrated similar in HPV positive and HPV negative cell lines in terms of patterns of upregulation of hypoxia induced genes in response to hypoxia. ${ }^{59}$ To this regard, Sorensen et al tried to find a relation, looking to the radiosensitivity, the impact of hypoxia and the effect of nimorazole in HPV positive and HPV negative cell lines which were irradiated with various doses under normoxia, hypoxia or hypoxia $+1 \mathrm{mM}$ nimorazole. ${ }^{60}$ As a result, although the HPV positive cell lines were up to 2.4 times more radiation sensitive than HPV negative cell lines, all HPV positive cells displayed the same response to hypoxia in radiosensitivity, with an OER in the range 2.3-2.9, with a sensitizer effect of nimorazole of 1.13-1.29, similar to HPV negative cells. It was also found that the hypoxic fraction of the HPV-positive tumor models decreased in response to radiotherapy. This trend was not seen in the HPV-negative tumors probably due to reoxygenation effect following irradiation. Authors concluded that HPV positive patients do not seem to benefit from nimorazole treatment as a consequence accounted for by the overall higher radiosensitivity of HPV positive cells. By these findings, future treatment directions for HPV-related oropharyngeal cancers should be based on radiobiological rationale accounting these properties as aimed by Marcu in a review. ${ }^{61}$

\section{Methods in Tumors Hypoxia Assessment}

Improvements have been achieved to assess hypoxia in HNSCC with direct and indirect methods. The most common direct method using an invasive technique consists of the polarographic electrodes approach in which the current between the cathode and the reference electrode has been found directly proportional to tissue $\mathrm{pO} 2 .{ }^{62}$ Among indirect methods, immunohistochemical staining of hypoxic cells as the angiogenesis assessment or the DNA strand break assay (comet assay) after radiation have been also accounted. ${ }^{63}$ Nowadays, advances in imaging and functional non invasive techniques provide a better mean for tumor hypoxia investigations. These techniques like the blood oxygen level-dependent magnetic resonance imaging (BOLD MRI) and PET imaging using hypoxiaspecific radiotracers have been found highly effective to record the heterogeneous distribution of oxygen within the tumor and its sub-volumes.

\section{The MRI Approach}

BOLD MRI uses the paramagnetic deoxyhemoglobin as a contrast agent to target RBCs because this method is sensitive to $\mathrm{pO} 2$ fluctuation in blood vessels. Several studies have been conducted to evaluate the relationship between pO2 and BOLD signal changes. ${ }^{64}$ A particular MRI study applied to this concern is the so called Intrinsic Susceptibility MRI (IS-MRI). This approach is based on the quantitation of the transverse relaxation rate $\mathrm{R}_{2}{ }^{*}$. This parameter seems to be very sensitive to the concentration of paramagnetic deoxyhemoglobin within the tissues vessels. Given the high concentration of deoxygenated RBCs in tumor microcirculation, tumors exhibit a relatively fast $\mathrm{R}_{2} *$ to be easier distinguished from normal tissues. Variations in levels of paramagnetic deoxyhemoglobin have been associated to cycling hypoxia both in human $\mathrm{CAL}^{\mathrm{R}}$ xenografts and HNSCC patients on a 3T clinical MRI clinical scanner by a study of Panek et al. ${ }^{65}$ 
In this research, as signal processing, the $\mathrm{R}_{2}{ }^{*}$ maps were calculatedfor each time point and the volume transfer constant between blood plasma and extracellular extravascular space, $\mathrm{K}^{\text {trans }}$ were elaborated for each voxel. As a result, spontaneous $\mathrm{R}_{2}$ * fluctuations were detected in both categories and predominantly associated with regions of heterogeneous perfusion and hypoxia in the $\mathrm{CAL}^{\mathrm{R}}$ xenografts. In patients, $\mathrm{R}_{2} *$ fluctuations spatially correlated with regions of lymph nodes with low $\mathrm{K}^{\text {trans }}$ values, typically nearest the necrotic cores. The authors concluded that such fluctuations are predominantly associated with regions of impaired tumor vasculature and this finding may be predictive for a poorer outcome. However, the MRI technique does not allow direct measurement of tissue pO2. BOLD MRI seems to be a more sensitive measure of oxygen levels for perfusion-related hypoxia than for diffusion-dependent or chronic hypoxia.

\section{The PET Approach}

Of all the currently existing techniques for in vivo detection of hypoxia, positron emission tomography (PET) is the best validated and not invasive clinical approach. PET is a functional imaging technique, which measures the in vivo distribution of radio-labelled isotopes, after the injection of a radioactive contrast medium. For hypoxia imaging, PET uses hypoxia-specific radioisotopes which together with $18 \mathrm{~F}-$ FDG (Fluorodeoxyglucose) show a complete representation of hypoxia distribution in the tumour, identifying and quantifing the hypoxic sub-volumes with subsequent impact on hypoxiccustomized radiation treatment. ${ }^{63}$ Among the hypoxia-specific radioisotopes, the most used belongs to radio-labelled nitroimidazoles which are well known to bind selectively to hypoxic cells where they are reduced. ${ }^{66}$ The first labelled nitroimidazole compound used in hypoxia PET studies has been the $18 \mathrm{~F}-$ MISO (18F-Fluoromisonidazole). Pretreatment 18F-MISO uptake has been found as an independent prognostic indicator following treatment of head and neck cancer. In fact, an high F-MISO uptakes seems to correspond to low tissue oxygen concentrations, which usually are indicative of poor response to treatment. ${ }^{67}$ Another interesting radiotracer which has been intensively studied in relation to hypoxia is 18F-FAZA (Fluoro-azomycin-arabinoside). This tracer show an advantage consisting of higher tumor to background and tumor-to-blood ratios due to more rapid clearance from blood and no-target tissues. ${ }^{68}$ While the 18 F-FDG is not particularly useful for the assessment of hypoxia with static PET imaging, in dynamic modality, it seems to offer a better valuable quantitative information on blood perfusion and also on drug pharmacokinetics through an indirect evaluation of vascular density and functionality. ${ }^{69}$ This approach is a work in progress. Although more expertise is needed for dynamic PET image acquisition and interpretation, with this method there is the possibility of following over time the radiotracer's metabolism in the region of interest. This allows for a better differentiation among metabolically dissimilar areas, including differently oxygenated regions. Further it provides data on vascular alterations during the radiotherapy. ${ }^{70}$ In line with these discoveries, a PET-based quantification of statistical properties of hypoxic tumor sub-volumes in HNSCC and their impact on failure rate of standard chemoradiation has been assessed by Chirla et al. As a result, the number and the intensity of hypoxic voxels significantly reduced during therapy. ${ }^{71}$ The change of the hypoxic volume during therapy, as well as the statistical distribution of the hypoxic fraction (HF) were evaluated. Interestingly the HF strongly correlated with the median T/M (tumor-to-muscle) SUV (standard up take value) ratio, but not with gross tumor volume. Hypoxia appeared 2.2 times more frequently in $\mathrm{T} 3+\mathrm{T} 4$ than in $\mathrm{T} 1+\mathrm{T} 2$ primary tumors. Authors concluded that the use of computational models with functional assessment of hypoxic regions could be effective in targeting radioresistant hypoxic tumor sub-volumes using complex dose gradients or even simpler boost doses treatment. Work is in progress.

\section{Hypoxia Studies in HNSCC}

Several studies have investigated hypoxia in HNSCC. Nordsmark et al found that patients with LRF presented a higher median hypoxic fraction than those with locoregional tumor control (22\% versus $6 \%$ ). A lower rate of actuarial two-year tumor control was recorded in patients with pretreatment median hypoxic fractions (defined as \% pO2 values $<2.5 \mathrm{mmHg})>15 \%$ than in those with fractions $\leq 15 \%$ (33\% and $77 \%$, respectively; $p=0.01) .{ }^{5}$ The fact that in normal tissue the median estimated $\mathrm{pO} 2$ values are 51 $\mathrm{mmHg}$ in subcutis and $37 \mathrm{mmHg}$ in skeletal muscle, and that these values remain relatively constant over $\mathrm{Hb}$ levels in the range of $10-16 \mathrm{~g} / \mathrm{dL}$, lends support to the idea that treatment outcome should be better for well-oxygenated tumors than for poorly oxygenated ones. In this regard, Brizel et al compared radiation therapy outcomes between well-oxygenated or poorly oxygenated primary tumors and metastatic lymph nodes in HNSCC patients, and found that tumor hypoxia with median $\%$ pO2 values $<10 \mathrm{mmHg}$ adversely affected two-year locoregional control ( $30 \%$ vs $73 \%, p=0.01$ ), DFS rate $(26 \%$ vs $73 \%, p=0.005)$, and OS rate $(35 \%$ vs $83 \%$, $p=0.02) .{ }^{6}$ A subsequent study evaluated the repeatability and prognostic impact of pretreatment pO2 histography on 
survival in patients with advanced head and neck cancer treated with accelerated hyperfractionated radiation therapy, with or without chemotherapy. Multivariate analysis indicated that the fraction with $\mathrm{pO} 2$ values $\leq 2.5 \mathrm{mmHg}$ was the only significant $(p=0.05)$ prognostic factor for survival, ${ }^{72}$ implying that the hypoxic fraction is a negative prognosticator for survival.

\section{Hypoxia and the Tumor Microenvironment}

As a direct effect, hypoxia may induce tumor resistance to radiation therapy and some chemotherapeutic agents via deprivation of molecular oxygen during treatment that requires adequate levels of intratumoral oxygen. Indirectly, hypoxia may lead to treatment resistance through modulation of gene expression and post-transcriptional or posttranslational modifications. The results include proteomic alterations that subsequently lead to changes in proliferation kinetics, cell-cycle position, or ratio of cells remaining in the G0 phase. These effects can influence the number of cells destroyed by either radiation therapy or chemotherapy. ${ }^{73}$ Hypoxia-induced proteomic changes may also influence cellular metabolism, such as the induction of the glycolytic pathway, or, for example, increased transcription of glucose transporters, DNA repair enzymes, growth factors, and proteins involved in tumor cell detachment, invasiveness, and resistance. Numerous hypoxia-inducible genes are controlled by the HIF1A and HIF2A transcription factors, including those involved in erythropoiesis, angiogenesis, glycolytic metabolism, and tumor invasiveness. ${ }^{74}$ HIF transcription factors are heterodimeric proteins that are upregulated in response to hypoxia. The major function of these proteins in tumor cells is to regulate hypoxia-driven changes in the expression of CA-9, GLUT-1, EPO, and other tumor growthpromoting factors. CA-9 is a transmembrane protein belonging to a large family of zinc metalloenzymes that catalyze the reversible hydration of carbon dioxide, and is involved in various biological processes, including acid-base balance and formation of saliva, cerebrospinal fluid, and gastric acid, and may also be involved in cell proliferation. CA-9 is also considered a prognostic marker for HNSCC, and its expression seems to correlate with poor survival, independently of tumor hypoxia. ${ }^{75}$ Lactate production may also have a role in hypoxia, as, in the absence of oxygen, glucose is converted to lactate through anaerobic glycolysis (Warburg effect). ${ }^{76}$ An increased HIF1A level is associated with increased glucose consumption, which requires increased levels of glucose transporters, such as GLUT-1, to upregulate glucose uptake. In turn, an increased level of GLUT-1 correlates with poor outcome for HNSCC patients as it decreases apoptosis, thereby favouring tumor growth. ${ }^{77}$ Tumor hypoxic tissue is acidified through lactate production, which increases tumor invasiveness and leads to metastasis. An increase in lactate due to increased glycolysis can be used as a biomarker in measuring tumor hypoxia. ${ }^{78}$

\section{The HIF Pathway in HNSCC Chemoradiation Resistance}

Solid tumors, such as HNSCC, present nonhomogeneous regions of hypoxia, the degree of which can be quantified through the expression of hypoxia-regulated proteins such as HIF1A and its downstream effectors, including CA-9. ${ }^{17}$ High levels of these proteins have been found to be strictly related to decreased survival in HNSCC patients treated with CCRT. Koukourakis et al found that high expression of HIF1A and HIF2A was associated with incomplete responses to chemoradiation ( $p=0.007$ and $p=0.02$, respectively). Univariate analysis indicated that high HIF1A and HIF2A expression was significantly associated with poor local relapse-free survival ( $p=0.003$ and $p=0.003$, respectively) and poor OS ( $p=0.05$ and 0.001 , respectively), while multivariate models showed that HIF2A expression was an independent prognostic factor. ${ }^{79}$ Based on these results, the authors conducted a new investigation to confirm the effect of hypoxia-regulated proteins on the outcome in platinumbased CCRT. Complete response was obtained in $85.2 \%$ of patients and the HIF1A level was marginally related to disease persistent after RT $(p=0.05)$. The level of HIF1A was significantly associated with poor local relapse-free survival (LRFS) $(p=0.006)$ and OS $(p=0.008)$, whereas HIF2A was not. Moreover, a significant association between CA-9 expression and poor LRFS was noted ( $p=0.01)$. This study confirmed that high levels of the hypoxia-regulated proteins HIF1A and CA-9 in HNSCC are predictors of resistance to platinum-based radiochemotherapy. ${ }^{18}$

\section{VEGF and Tumor EPOR Expression}

The role of VEGF in tumor oxygenation via neovascularization remains undisputed, and is associated with hypoxia-related signaling pathways. During hypoxia, HIF1A is known to upregulate the expression of VEGF, thereby inducing neoangiogenesis. ${ }^{80}$ Increased VEGF production by tumor cells is associated with poor prognosis, nodal metastasis, advanced clinical stage, and low survival 
in HNSCC. ${ }^{81}$ These observations, together with the finding that EPOR is expressed in several cancers, including HNSCC, ${ }^{14}$ have led to the suggestion that autocrine or paracrine EPO signaling, and, consequently, rHu-EPO treatment, may play a role in HNSCC malignant progression and treatment resistance.

\section{EPOR Expression in HNSCC}

Li et al investigated the expression of EPO and EPOR in 65 resected squamous cell carcinomas (SCC) of the tongue. $^{15}$ Their results showed that the proportion of changes in EPO and EPOR expression in these specimens was greater than that in normal squamous epithelium $(p<0.05)$. EPO expression was associated with age, microvessel density, and tumor stage $(p<0.05)$. After adjusting for other clinicopathological factors, EPO and EPOR expression remained independent, adverse prognosticators for postoperative survival $(p<0.05)$. The authors concluded that EPOR expression influences the prognosis of carcinogenesis, angiogenesis, and malignant progression of SCC of the tongue, suggesting that a hypoxic tumor microenvironment might stimulate angiogenesis and promote tumor survival and growth. In support of these findings, Lin et al analyzed 256 surgical specimens of patients who had not received previous radiotherapy and/or chemotherapy, and found that EPOR was widely overexpressed in oral squamous cancer tissues. These results indicated that elevated EPOR expression may be significantly correlated with advanced $\mathrm{T}$ classification $(p<0.001)$, advanced TNM stage $(p<0.001)$, and positive $\mathrm{N}$ classification $(p=0.001)$. Furthermore, univariate analysis indicated that patients with high tumor EPOR expression showed lower fiveyear $\operatorname{OS}(p=0.0011)$ and disease-specific survival rates $(p=0.0017)$ than patients exhibiting low tumor levels of EPOR. The EPO receptor was assessed to be an independent prognostic marker in patients with oral cancer. ${ }^{16}$

\section{EPO/EPOR Signaling and Anemia in HNSCC}

Autocrine or paracrine EPO signaling may also be activated by $\mathrm{rHu}$-EPO therapy in the malignant progression and invasiveness of HNSCC, and could potentially be the source of the detrimental effects evidenced in the main EPO trials. ${ }^{82}$ Mohyeldin et al investigated the expression and function of EPO and EPOR in HNSCC-derived biopsies and cell lines. The EPO responsiveness of the cell lines was assessed through epoetin alpha-induced tyrosine phosphorylation of
Janus kinase 2 (JAK2). The authors observed a significant association between disease progression and expression of EPO and its receptor in 32 biopsies. EPO expression was highest in malignant cells, particularly within hypoxic regions and regions of tumor infiltration. Although the expression of both EPO and EPOR was detected in human head and neck carcinoma cell lines, only the expression of EPOR was upregulated as a result of hypoxia. Epoetin alpha treatment induced marked JAK2 phosphorylation and enhanced cell invasion, while inhibition of JAK2 phosphorylation reduced both basal and EPO-induced invasiveness. Further, Winter et al also examined the relationship between EPO/EPOR and anemia. ${ }^{83}$ First, they found that EPO and EPOR were expressed in the cytoplasm of $90 \%$ and $95 \%$ of tumors, respectively, and also found a positive correlation between EPO and EPOR expression ( $r=0.18, p=0.03$ ). They then investigated the stimuli regulating the expression of both EPO and EPOR in vivo, as well as their functional significance. Their results showed that EPO, but not EPOR, colocalized with HIF1A and CA9, thereby providing further evidence that hypoxia is the main stimulus for EPO expression in tumors. The expression of HIF1A $(p<0.01)$ and CA-9 $(p=0.002)$ correlated with the expression of EPO, but not with that of EPOR, and no correlation was found between $\mathrm{Hb}$ levels and EPO $(r=0.07, p=0.36)$ or EPOR $(r=0.02, p=0.8)$ levels. No survival difference was detected between high or low EPO or EPOR expression ( $p=0.59$ and $p=0.98$, respectively). The authors concluded that, in HNSCC, the HIF pathway seems to regulate the expression of EPO, but not EPOR, at the mRNA level in vivo. Furthermore, according to these results, anemia does not seem to influence the hypoxic microenvironment of tumors enough to affect EPO expression. Thus, the authors provided strong evidence for the hypoxic regulation of EPO and a local EPO/EPOR autocrine signaling pathway in tumors.

\section{Conclusion: Who Drives This Carousel?}

Hypoxia, and not anemia, seems to be the driver of resistance to rHu-EPO treatment in HNSCC therapy. The EPO trial failures should be considered as the tip of an iceberg comprising the hypoxia-related HIF signaling pathway. Exogenous EPO may be acting via its receptor, EPOR, in tumor cells through the HIF pathway in vivo, thereby enhancing tumor growth and invasiveness. One solution to overcome this resistance could be the combined 
targeting of HIF and CA-9 proteins, which represents an important challenge in the development of new anticancer drugs. In this regard, several HIF inhibitors or those targeting the tumor-associated isoforms of CA-9 have been developed and are currently under investigation in clinical trials for cancer treatment. ${ }^{84}$ Although these compounds have shown promising anticancer effects in preclinical studies, substantial clinical trials have yet to be conducted. A clinical Phase I trial testing the CA-9 inhibitor, SLC0111 (also known as WBI-5111), as monotherapy for the treatment of solid tumors has recently been completed, but the results have not yet been published. ${ }^{85}$ SLC-0111 is a simple, ureido-substituted, benzenesulfonamide derivative which exhibits significant inhibitory activity against CA-9 and CA-12 in vitro. ${ }^{86}$ In vivo, SLC-0111 was shown to inhibit the growth of CA-9/12-expressing tumors, and promoted a notable depletion of the cancer stem cell population and reduced metastatic potential. Important antitumor effects have also been observed for SLC-0111 in combination with other anticancer agents used clinically, such as paclitaxel and doxorubicin. ${ }^{87}$ Although the results of the phase I clinical trial are not yet available, the compound has been scheduled for Phase II trials.

\section{Acknowledgment}

This review was supported by the Medical Oncology and Hematology staff.

\section{Disclosure}

The authors report no conflicts of interest in this work.

\section{References}

1. Anemia BHF. Harrison's Principles of Internal Medicine 10th Edition. McGraw Hill; 1983:54.

2. Hu K, Harrison LB. Impact of anemia in patients with head and neck cancer treated with radiation therapy. Curr Treat Options Oncol. 2005;6(1):31-35. doi:10.1007/s11864-005-0011-4

3. Kumar P. Impact of anaemia in patients with head and neck cancer. Oncologist. 2000;5(suppl 2):13-18. doi:10.1634/theoncologist.5suppl_2-13

4. Horsman MR, Wouters BJ, Joiner MC, Overgaard J. The oxygen effect and fractionated radiotherapy Ch 15.1. In: Joiner M, van der Kogel A, editors. Basic Clinical Radiobiology. 4th. Hodder Arnold editor; 2009:211-213.

5. Nordsmark M, Overgaard M, Overgaard J. Pretreatment oxygenation predicts radiation response in advanced squamous cell carcinoma of the head and neck. Radiother Oncol. 1996;41:31-39. doi:10.1016/ S0167-8140(96)91811-3

6. Brizel DM, Dodge RK, Clough RV, Dewhirst MW. Oxygenation of head and neck cancer: changes during radiotherapy and impact on treatment outcome. Radiother Oncol. 1999;53(2):113-117. doi:10.1016/S01678140(99)00102-4
7. Thews O, Koenig R, Kelleher DK, Kutzner J, Vaupel P. Enhanced radiosensitivity in experimental tumours following erythropoietin treatment of chemotherapy-induced anaemia. $\mathrm{Br} \quad \mathrm{J}$ Cancer. 1998;78:752-756. doi:10.1038/bjc. 1998.572

8. Stüben G, Pöttgen C, Knühmann K, et al. Erythropoietin restores the anemia-induced reduction in radiosensivity of experimental human tumors in nude mice. Int J Radiat Oncol Biol Phys. 2003;55:1358-1362. doi:10.1016/S0360-3016(03)00012-9

9. Stüben G, Thews O, Pöttgen C, et al. Impact of anemia prevention by recombinant human erythropoietin on the sensitivity of xenografted glioblastomas to fractionated irradiation. Strahlenther Onkol. 2003;179(9):620-625. doi:10.1007/s00066-003-1110-4

10. Henke M, Laszig R, Rübe C, et al. Erythropoietinto treat head and neck cancer patients with anaemia undergoing radiotherapy: randomised, double-blind, placebo-controlled trial. Lancet. 2003;362:1255-1260. doi:10.1016/S0140-6736(03)14567-9

11. Machtay M, Pajak TF, Suntharalingam M, et al. Radiotherapy with or without erythropoietin for anemic patients with head and neck cancer: A randomized trial of the Radiation Therapy Oncology Group (RTOG 99-03). Int J Radiat Oncol Biol Phys. 2007;69:1008-1017. doi:10.1016/j.ijrobp.2007.04.063

12. Hoskin PJ, Robinson M, Slevin N, Morgan D, Harrington K, Gaffney C. Effect of epoetin alfa on survival and cancer treatment-related anemia and fatigue in patients receiving radical radiotherapy with curative intent for head and neck cancer. $J$ Clin Oncol. 2009;27(34):5751-5756. doi:10.1200/JCO.2009.22.3693

13. Overgaard J, Hoff CM, Hansen HS, et al. DAHANCA 10 - Effect of darbepoetin alfa and radiotherapy in the treatment of squamous cell carcinoma of the head and neck. A multicenter, open-label, randomized, Phase 3 trial by the Danish head and neck cancer group. Int J Radiat Oncol Biol Phys. 2018;127(1):12-19.

14. Farrell F, Lee A. The erythropoietin receptor and its expression in tumor cells and other tissues. Oncologist. 2004;9:18-30. doi:10.1634/ theoncologist.9-90005-18

15. Li HG, Li JS, Chen WL, Wang L, Wu DH, Lin ZY. Prognostic significance of erythropoietin and erythropoietin receptor in tongue squamous cell carcinoma. $\mathrm{Br} \quad \mathrm{J}$ Oral Maxillofac Surg. 2009;47:470-475. doi:10.1016/j.bjoms.2009.06.001

16. Lin YT, Chuang $\mathrm{HC}$, Chen $\mathrm{CH}$, et al. Clinical significance of erythropoietin receptor expression in oral squamous cell carcinoma. BMC Cancer. 2012;12:194. doi:10.1186/1471-2407-12-194

17. Beasley NJ, Wykoff CC, Watson PH, et al. Carbonic anhydrase IX, an endogenous hypoxia marker, expression in head and neck squamous cell carcinoma and its relationship to hypoxia, necrosis, and microvessel density. Cancer Res. 2001;61(13):52625267.

18. Koukourakis MI, Giatromanolaki A, Danielidis V, Sivridis E. Hypoxia inducible factor (HIF1alpha and HIF2alpha) and carbonic anhydrase 9 (CA9) expression and response of head-neck cancer to hypofractionated and accelerated radiotherapy. Int $J$ Radiat Oncol Biol Phys. 2008;84(1):47-52. doi:10.1080/09553000701616114

19. Caro JJ, Salas M, Ward A, Goss G. Anemia as an independent prognostic factor for survival in patients with cancer: a systemic, quantitative review. Cancer. 2001;91:2214-2221. doi:10.1002/(ISSN) 1097-0142

20. Littlewood TJ. The impact of hemoglobin levels on treatment outcomes in patients with cancer. Semin Oncol. 2001;28(2 Suppl 8):49-53. doi:10.1016/S0093-7754(01)90213-1

21. Roxburgh CS, McMillan DC. Role of systemic inflammatory response in prediciting survival in patients with primary operable cancer. Future Oncol. 2010;1:149-163. doi:10.2217/fon.09.136

22. Bohlius J, Weingart O, Trelle S, Engert A. Cancer-related anemia and recombinant human erythropoietin-an updated overview. Nat Clin Pract Oncol. 2006;3(3):152-164. doi:10.1038/ncponc0451

23. Spivak JL. The anaemia of cancer: death by a thousand cuts. Nat Rev Cancer. 2005;5:543-555. doi:10.1038/nrc1648 
24. Ludwig H, Van Belle S, Barrett-Lee P, et al. The European Cancer Anaemia Survey (ECAS): a large multinational, prospective survey defining the prevalence, incidence, and treatment of anaemia in cancer patients. Eur J Cancer. 2004;40(15):2293-2306. doi:10.1016/j.ejca.2004.06.019

25. Groopman JE, Itri LM.Chemotherapy-induced anemia in adults: incidence and treatment. J Natl Cancer Inst. 1999;91:1616-1623. doi:10.1093/jnci/91.19.1616

26. Dasari S, Tchounwou PB. Cisplatin in cancer therapy: molecular mechanisms of actions. Eur J Pharmacol. 2014;740(5):364-378. doi:10.1016/j.ejphar.2014.07.025

27. Pignon JP, Bourthis J, Domenge C, Designé I. Chemotherapy added to locoregional treatments for head and neck squamous-cell carcinoma: three meta-analyses of updated individual data. MACH-NC Collaborative Group. Meta-analysis of chemotherapy on head and neck cancer. Lancet. 2000;355:949-955. doi:10.1016/S0140-6736(00)90011-4

28. Pignon JP, le Maïtre A, Maillard E, Bourthis J; MACH-NC Collaborative Group. Metanalysis of chemotherapy in head and neck cancer (MACH-NC): an update on 93randomised trials and 17,346 patients. Radiother Oncol. 2009;92:4-14. doi:10.1016/j.radonc.2009.04.014

29. Forastiere A, Goepfert H, Maor M, et al. Concurrent chemotherapy and radiotherapy for organ preservation in advanced laryngeal cancer. N Engl J Med. 2003;349:2091-2098. doi:10.1056/NEJMoa031317

30. Ang KK, Chen A, Curran WJ Jr, et al. Head and neck carcinoma in the United States: first comprehensive report of the Longitudinal Oncology Registry of Head and Neck Carcinoma (LORHAN). Cancer. 2012;118:5783-5792. doi:10.1002/cncr.v118.23

31. Strojan P, Vermorken JB, Beitler JJ, et al. Cumulative cisplatin dose in concurrent chemoradiotherapy for head and neck cancer: a systematic review. Head Neck. 2016;38(1):2151-2158. doi:10.1002/hed.24026

32. Hanigan MH, Devarajan P. Cisplatin nephrotoxicity: molecular mechanisms. Cancer Ther. 2003;1:47-61.

33. Wood PA, Hrushesky JM. Cisplatin -associated anemia: an Erythropoietin deficiency syndrome. J Clin Invest. 1995;95:1650-1659. doi:10.1172/JCI117840

34. Fortin A, Wang CS, Vigneault E. Effect of pretreatment anemia on treatment outcome of concurrent radiochemotherapy in patients with head and neck cancer. Int J Radiat Oncol Biol Phys. 2008;72 (1):255-260. doi:10.1016/j.ijrobp.2008.04.079

35. Overgaard J, HS H, Overgaard M, et al. A randomized double-blind Phase III study of nimorazole as a hypoxic radiosensitizers of primary radiotherapy in supreglottic larynx and pharynx carcinoma. Results of the Danish Head and Neck Cancer Study (DAHANCA) Protocol 5-85. RadiotherOncol. 1998;46:136-146.

36. Fein DA, Lee WR, Hanlon AL, et al. Pretreatment hemoglobin level influences local control and survival of T1-T2 squamous cell carcinomas of the glottic larynx. J Clin Oncol. 1995;13(8):2077-2083. doi:10.1200/JCO.1995.13.8.2077

37. Prosnitz RG, Yao B, Farrell CL, Clough R, Brizel DM. Pretreatment anemia is correlated with the reduced effectiveness of radiation and concurrent chemotherapy in advanced head and neck cancer. Int J Radiat Oncol Biol Phys. 2005;61(4):1087-1095. doi:10.1016/j. ijrobp.2004.07.710

38. Rades D, Stoehr M, Kazic N, et al. Locally advanced stage IV squamous cell carcinoma of the head and neck: impact of pre-radiotherapy hemoglobin level and interruption during radiotherapy. Int J Radiat Oncol Biol Phys. 2008;70(4):1108-1114. doi:10.1016/j.ijrobp.2007.07.2380

39. Narayanaswamy RK, Potharaju M, Vaidhyswaran AN, Perumal K. Pre-radiotherapy haemoglobin level is a prognosticator in locally advanced head and neck cancers treated with concurrent chemoradiation. J Clin Diagn Res. 2015;9(6):14-18.

40. Su NW, Liu CJ, Leu YS, Lee JC, Chen YJ, Chang Y. Prolonged radiation time and low nadir hemoglobin during postoperative concurrent chemoradiotherapy are both poor prognostic factors with synergistic effect on locally advanced head and neck cancer patients. OncoTargetsTher. 2015;8:251-258.
41. Aapro MS, Linkb H. September 2007 update on EORTC guidelines and anemia management with erythropoiesis-stimulating agents. Oncologist. 2008;13(suppl 3):33-36. doi:10.1634/theoncologist.13-S3-33

42. Bohlius J, Bohlke K, Castelli R, et al. Management of cancer-associated anemia with erythropoiesis-stimulating agents: ASCO/ASH clinical practice guideline update. Blood Adv. 2019;3 (8):1197-1210. doi:10.1182/bloodadvances.2018030387

43. Oster W, Herrmann F, Gamm H, et al. Erythropoietin for the treatment of anemia of malignancy associated with neoplastic bone marrow infiltration. J Clin Oncol. 1990;8:956-962. doi:10.1200/JCO.1990.8.6.956

44. Koury MJ, Bondurant MC. Erythropoietin retards DNA breakdown and prevents programmed death in erythroid progenitor's cells. Science. 1990;248:378-381. doi:10.1126/science.2326648

45. Koury ST, Bondurant MC, Koury MJ. Localization of erythropoietin synthesizing cells in murine kidneys by in situ hybridization. Blood. 1988;71(2):524-527. doi:10.1182/blood.V71.2.524.524

46. Silver DF, Piver MS. Effects of recombinant human erythropoietin on the antitumor effect of cisplatin in SCID mice bearing human ovarian cancer: a possible oxygen effect. Gynecol Oncol. 1999;73:280-284. doi:10.1006/gyno. 1999.5368

47. Zips D. Tumor growth and response to radiation.Ch 7.3. In: Joiner M, van der Kogel A, editors. Basic Clinical Radiobiology. 4th ed. Hodder Arnold editor; 2009:92-98.

48. Bayer C, Vaupel P. Acute versus chronic hypoxia in tumors: controversial data concerning time frames and biological consequences. Strahlenther.Onkol. 2012;188(7):616-627. doi:10.1007/s00066-0120085-4

49. Isa AY, Ward TH, West CM, Slevin NJ, Homer JJ. Hypoxia in head and neck cancer. Br J Radiol. 2006;79(946):791-798. doi:10.1259/ bjr/17904358

50. Harrison LB, Chadha M, Hill RJ, Hu K, Shasha D. Impact of tumor hypoxia and anemia on radiation therapy outcomes. Oncologist. 2002;7(6):492-508. doi:10.1634/theoncologist.7-6-492

51. Bayer C, Shi K, Astner ST, Maftei CA, Vaupel P. Acute versus chronic hypoxia: why a simplified classification is simply not enough. Int J Radiat Oncol Biol Phys. 2011;80:965-968. doi:10.1016/j. ijrobp.2011.02.049

52. D'Souza G, Kreimer AR, Viscidi R, et al. Case-control study of human papillomavirus and oropharyngeal cancer. $N$ Engl $J$ Med. 2007;356:1944-1956. doi:10.1056/NEJMoa065497

53. Lassen P. The role of Human papillomavirus in head and neck cancer and the impact on radiotherapy outcome. Radiother Oncol. 2010;95:371-380. doi:10.1016/j.radonc.2010.04.022

54. Toustrup K, Sorensen BS, Lassen P, Wiuf C, Alsner J, Overgaard J. Gene expression classifier predicts for hypoxic modification of radiotherapy with nimorazole in squamous cell carcinomas of the head and neck. Radiother Oncol. 2012;102:122-129. doi:10.1016/j.radonc.2011.09.010

55. Kong CS, Narasimhan B, Cao H, et al. The relationship between human papillomavirus status and other molecular prognostic markers in head and neck squamous cell carcinomas. Int J Radiat Oncol Biol Phys. 2009;74:553-561. doi:10.1016/j.ijrobp.2009.02.015

56. Lassen P, Eriksen JG, Hamilton-Dutoit S, Tramm T, Alsner J, Overgaard J. HPV-associated p16-expression and response to hypoxic modification of radiotherapy in head and neck cancer. Radiother Oncol. 2010;94:30-35. doi:10.1016/j.radonc.2009.10.008

57. Rischin D, Young RJ, Fisher R, et al. Prognostic significance of p16INK4A and human papillomavirus in patients with oropharyngeal cancer treated on TROG 02.02 phase III trial. J Clin Oncol. 2010;28:4142-4148. doi:10.1200/JCO.2010.29.2904

58. Arenz A, Ziemann F, Mayer C, et al. Increased radiosensitivity of HPV-positive head and neck cancer cell lines due to cell cycle dysregulation and induction of apoptosis. Strahlenther Onkol. 2014;190:839-846. doi:10.1007/s00066-014-0605-5

59. Sorensen BS, Busk M, Olthof N, et al. Radiosensitivity and effect of hypoxia in HPV positive head and neck cancer cells. Radiother Oncol. 2013;108:500-505. doi:10.1016/j.radonc.2013.06.011 
60. Sorensen BS, Busk M, Horsman MR, et al. Effect of radiation on cell proliferation and tumor hypoxia in HPV-positive head and neck cancerin vivomodels. Anticancer Res. 2014;34::6297-6304.

61. Marcu LG. Future treatment directions for HPV-associated head and neck cancer based on radiobiological rationale and current clinical evidence. Crit Rev Oncol Hematol. 2016;103:27-36. doi:10.1016/j. critrevonc.2016.05.002

62. Carreau A, Hafny-Rahbi BE, Matejuk A, Grillon C, Kieda C. Why is the partial oxygen pressure of human tissues a crucial parameter? Small molecules and hypoxia. J Cell Mol Med. 2011;15 (6):1239-1255. doi:10.1111/j.1582-4934.2011.01258.x

63. Marcu LG, Harriss-Phillips WM, Filip SM. Hypoxia in head and neck cancer in theory and practice: a PET-based imaging approach. Comput Math Methods Med. 2014;2014:624642.

64. Hallac RR, Zhou H, Pidikiti R, et al. Correlations of noninvasive BOLD and TOLD MRI with $\mathrm{pO} 2$ and relevance to tumour radiation response. Magn Reason Med. 2014;71(5):1863-1873. doi:10.1002/ mrm. 24846

65. Panek R, Welsh L, Lauren CJ, et al. Non-invasive imaging of cycling hypoxia in head \& neck cancer using intrinsic susceptibility MRI. Clin Cancer Res. 2017;23(15):4233-4241. doi:10.1158/1078-0432. CCR-16-1209

66. Nunn A, Linder K, Strauss HW. Nitroimidazoles and imaging hypoxia. Eur J Nucl Med. 1995;22(3):265-280. doi:10.1007/ BF01081524

67. Rajendran JG, Schwart DL, O’Sullivan J, et al. Tumor hypoxia imaging with [F-18]fluoromisonidazole positron emission tomography in head and neck cancer. Clin Cancer Res. 2006;12 (18):5435-5441. doi:10.1158/1078-0432.CCR-05-1773

68. Sorger D, Patt M, Kumar P, et al. [18F]Fluoroazomycinarabinofuranoside (18FAZA) and [18F]Fluoromisonidazole (18FMISO): a comparative study of their selective uptake in hypoxic cells and PET imaging in experimental rat tumors. Nucl Med Biol. 2003;30(3):317-326. doi:10.1016/S0969-8051(02)00442-0

69. Strauss LG, Pan L, Cheng C, Haberkorn UA, DimitrakopoulouStrauss A. Shortened acquisition protocols for the quantitative assessment of the2-tissue-compartment model using dynamic PET/CT 18F-FDG studies. J Nucl Med. 2011;52(3):379-385. doi:10.2967/ jnumed.110.079798

70. Rodal J, Rusten E, Søvik A, Skogmo HK, Malinen E. Functional imaging to monitor vascular and metabolic response in canine head and neck tumors during fractionated radiotherapy. Acta Oncol. 2013;52(7):1293-1299. doi:10.3109/0284186X.2013.812800

71. Chirla R, Marcu LG. PET-based quantification of statistical properties of hypoxic tumor subvolumes in head and neck cancer. Phys Med. 2016;32(1):23-35. doi:10.1016/j.ejmp.2015.12.006

72. Rudat V, Stadler P, Becker A, et al. Predictive value of the tumor oxygenation by means of pO2 histography in patients with advanced head and neck cancer. Strahlenther Onkol. 2001;177(9):462-468. doi:10.1007/PL00002427
73. Vaupel P, Harrison I. Tumor hypoxia: causative factors, compensatory mechanisms, and cellular response. Oncologist. 2004;9(5):4-9. doi:10.1634/theoncologist.9-90005-4

74. Shih SC, Claffey KP. Role of AP-1 and HIF-1 transcription factors in TGF-beta activation of VEGF expression. Growth Factors. 2001;19 (1):19-34. doi:10.3109/08977190109001073

75. Peridis S, Pilgrim G, Athanasopoulos I, Parpounas K. Carbonic anhydrase-9 expression in head and neck cancer: a meta-analysis. Eur Arch Otorhinolaryngol. 2011;268(5):661-670. doi:10.1007/ s00405-011-1488-z

76. Koppenol WH, Bounds PL, Dang CV. Otto Warburg's contributions to current concepts of cancer metabolism. Nat Rev Cancer. 2011;11 (5):325-337. doi:10.1038/nrc3038

77. Li S, Yang X, Wang P, Ran X. The effects of GLUT1 on the survival of head and neck squamous cell carcinoma. Cell Physiol Biochem. 2013;32(3):624-634.

78. Walenta S, Wetterling M, Lehrke M, et al. High lactate levels predict likelihood of metastases, tumor recurrence, and restricted patient survival in human cervical cancers. Cancer Res. 2000;60(4):916-921.

79. Koukourakis MI, Giatromanolaki A, Sivridis E, et al. Hypoxiainducible factor (HIF1A and HIF2A), angiogenesis, and chemoradiotherapy outcome of squamous cell head-and-neck cancer. Int J Radiat Oncol Biol Phys. 2002;53:1192-1202. doi:10.1016/ S0360-3016(02)02848-1

80. Ferrara N, Gerber HP, LeCouter J. The biology of VEGF and its receptors. Nat Med. 2003;9:669-676. doi:10.1038/nm0603-669

81. Kyzas PA, Stefanou D, Batistatou A, Agnantis NJ. Hypoxia-induced tumor angiogenic pathway in head and neck cancer: an in vivo study. Cancer Lett. 2005;225(2):297-304. doi:10.1016/j.canlet.2004.11.060

82. Mohyeldin A, Lu H, Dalgard C, et al. Erythropoietin signalling promotes invasiveness of human head and neck squamous cell carcinoma. Neoplasia. 2005;7:537-543. doi:10.1593/neo.04685

83. Winter SC, Shah KA, Campo L, et al. Relation of erythropoietin and erythropoietin receptor expression to hypoxia and anemia in head and neck squamous cell carcinoma. Clin Cancer Res. 2005;11 (21):7614-7620. doi:10.1158/1078-0432.CCR-05-1097

84. Supuran CT, Winum JY. Carbonic anhydrase IX inhibitors in cancer therapy: an update. Future Med Chem. 2015;7(11):1407-1414. doi: $10.4155 /$ fmc. 15.71

85. A Phase I, multi-center, open-label, study to investigate the safety, tolerability and pharmacokinetic of SLC-0111 in subjects with advanced solid tumours. 2016. Available from: https://clinicaltrials. gov/ct2/show/NCT02215850. Accessed September 15, 2017).

86. Carta F, McDonald PC, Lou YET, et al. Ureido-substituted benzenesulfonamides potently inhibit carbonic anhydrase IX and show antimetastatic activity in a model of breast cancer metastasis. $J$ Med Chem. 2011;54:1896-1902. doi:10.1021/jm101541x

87. Lou Y, McDonald PC, Oloumi A, et al. Targeting tumor hypoxia: suppression of breast tumor growth and metastasis by novel carbonic anhydrase IX inhibitors. Cancer Res. 2011;71:3364-3376. doi:10.1158/0008-5472. CAN-10-4261
OncoTargets and Therapy

\section{Publish your work in this journal}

OncoTargets and Therapy is an international, peer-reviewed, open access journal focusing on the pathological basis of all cancers, potential targets for therapy and treatment protocols employed to improve the management of cancer patients. The journal also focuses on the impact of management programs and new therapeutic

Submit your manuscript here: https://www.dovepress.com/oncotargets-and-therapy-journa agents and protocols on patient perspectives such as quality of life, adherence and satisfaction. The manuscript management system is completely online and includes a very quick and fair peer-review system, which is all easy to use. Visit http://www.dovepress.com/ testimonials.php to read real quotes from published authors. 